\title{
Determination of Exercise Intensity that Elicits Maximal Fat Oxidation on a Body Weight Supported Treadmill in Normal Weight Males
}

\author{
Toni T. LaSala \\ Jordan Cola \\ Michael A. Figueroa \\ Racine Emmons \\ Department of Kinesiology \\ William Paterson University \\ 300 Pompton Rd. Wayne, NJ 07470, USA
}

\begin{abstract}
Purpose: To determine the exercise intensity that elicits maximal fat oxidation while walking at $100 \%$ to $75 \%$ on a lower body positive pressure treadmill (LBPP) in normal weight men. Results: Peak oxygen consumption $\left(\dot{V} O_{2 p e a k}\right)$ was significantly lower $(p<.001)$ at $75 \% \mathrm{BW}$ compared to $100 \% \mathrm{BW}$ where fat oxidation $(F O)$, and respiratory exchange ratio (RER) were not significantly lower ( $p>.05$ ) at $75 \%$ BW compared to $100 \% B W$. Conclusion: Unweighting on the LBPP can be used as a low to moderate intensity exercise intervention for normal weight individuals by reducing force, improving exercise tolerance, as well as maintaining cardio respiratory demand.
\end{abstract}

Keywords: lower body positive pressure treadmill, peak fat oxidation, peak oxygen consumption, respiratory exchange ratio.

\section{Introduction}

Exercise intensity is an important regulator in carbohydrate (CHO) and fat utilization (Romijn et al., 1993, Achten \& Jeukendrup, 2003) and may have important implications for exercise training. The more intense the activity, the greater the contribution of $\mathrm{CHO}$ oxidation to energy expenditure (Brooks \& Mercier, 1994) however, during low to moderate exercise intensity absolute fat oxidation rates (grams per minute) will increase then decline at higher intensities (Takagi, Sakamoto, Midorikawa, Konishi \& Katsumura, 2014). The exercise intensity that elicits the highest fat oxidation rate has been termed Lipoxmax (Perez-Martin \& Mercier, 2001), Fatoxmax (O’Deriaz et al., 2001; Nordby et al., 2006) or Fatmax (Achten, Gleeson, \& Jeukendrup, 2002; Jeukendrup, 2003 \& Venables, Achten, \& Jeudendrup, 2005) and peak fat oxidation (PFO) (Bogdanis, Vangelakoudi, \& Maridaki, 2008).

The balance of carbohydrate and lipid utilization during exercise was summarized by a physiological theory of exercise called the "crossover concept" (Brooks \& Mercier 1994). This concept implies that the predominant fuel source oxidized in an individual at rest and low exercise intensities are lipids, and at higher intensities, the predominant fuel source oxidized is carbohydrates . The crossover point (approximately $50 \% \mathrm{~V}_{2 \text { peak }}$ ) indicates that as exercise intensity increases, there is a shift from fat utilization to carbohydrate utilization where carbohydrates eventually becomes the predominant fuel at $>70 \% \dot{\mathrm{V}_{2 p e a k}}$ (Brun Varlet-Marie, Romain \& Mercier, 2012). Furthermore, the absolute rate of fat oxidation becomes negligible at higher intensities (Achten, et al., 2002). A reduction in fat oxidation has been related to changes in body weight or body composition (Zurlo et al., 1990) whereas a high capacity to oxidize fat may be a benefit for maintaining body composition.

The crossover point is also dependent upon the effects of exercise intensity, nutritional status, gender, age, overtraining and previous exercise experience. Venables et al., (2005) found that during an incremental treadmill protocol, healthy men and women had a mean maximal fat oxidation of $0.46 \pm 0 \mathrm{~g} / \mathrm{min}$ which ranged from 0.18 $\mathrm{g} / \mathrm{min}$ to $1.01 \mathrm{~g} / \mathrm{min}$ which demonstrated the five-fold variability. The "crossover concept", (Brooks \& Mercier, 1994) has generated interest in developing exercise protocols and testing for the purpose of assessing this balance of substrates (Brun et al., 2013). 
Measuring substrate utilization using indirect calorimetry on a cycle ergometer, Romijn et al., (1993) found fat oxidation increased from $25 \%$ to $65 \%$ of $\dot{\mathrm{VO}}_{2 \text { peak }}$ and declined again at $85 \%$. In their study, they only investigated three exercise intensities, $\left(25 \%, 65 \%\right.$ and $85 \%$ of $\left.\dot{\mathrm{VO}}_{2 \text { peak }}\right)$ and since the difference between intensities was large, fat oxidation rates may not be accurate. In another study (Achten et al., 2002), a protocol was developed to determine an intensity to elicit maximal fat oxidation rates with a larger number of exercise intensities and smaller increments. Their results show that PFO rates occurred at $64 \% \dot{\mathrm{VO}}_{2 \text { peak }}$, which is in agreement with the results of Romijn et al., (1993).

Exercise training and substrate utilization is well documented in normal weight individuals (Jeukendrup et al., 1997; Kanaley, Weatherup-Dentes, Alvarado, \& Whitehead, 2001; van Loon. Jeukendrup, Saris, \& agenmakers, 1999), where fat is the preferred energy source at rest and low to moderate intensity exercise of long duration (Brooks \& Mercer 1994; Romijn et al., 1993; Thompson et al., 1998). Sial, Coggan, Hickner, \& Klein, (1999) demonstrated that exercise training in elderly subjects increased total fat oxidation without a change in lipolysis or FFA availability. Similar data were found in obese and healthy lean subjects using a low-intensity exercise program for only a few hours per week (Schrauwen et al., 2002; van Aggel- Leijssen et al., 2002), and might be more appropriate for (obese) insulin-resistant subjects. Exercise is an effective means to improve insulin sensitivity in lean, obese and diabetic individuals and when performed on a regular basis can produce long-term changes within skeletal muscle (Venables \& Jeukendrup, 2008).

Very few studies have looked at peak fat oxidation rates during walking (Achten et al., 2003; Venables et al., 2005; 2008). The majority of the studies used cycling as the type of exercise (Achten \& Jeukendrup, 2003; 2004; Perez-Martin et al., 2001) where fat oxidation is approximately $30 \%$ lower on the cycle compared to walking at an equivalent intensity (Achten et al., 2003). Those studies used walking speeds that were between 6.5 and $7.5 \mathrm{~km} / \mathrm{h}$, which is close to the transition of walking to running. Furthermore, no study has looked at PFO rates during walking on a weight-supported treadmill in normal weight individuals.

Partial body weight support treadmill training (PBWSTT) devices have emerged as a rehabilitation and training tool. These devices have been used as training protocols for neurological and orthopedic rehabilitation to assist in upright ambulation by reducing biomechanical risks by decreasing ground reaction forces (GRFs) in specific populations (American Physiological Society, 2005). Training on a PBWSTT device, requires the individual to wear a modified climbing harness around the waist and pelvis, which is attached to a weight suspension apparatus where a constant upward force on the body is applied. This type of device cannot only be cumbersome and uncomfortable but can inhibit circulation in extended rehabilitation and training sessions (Griffin Tolani, \& Kram, 1999; Grabowski, Farley, \& Kram, 2005).

To determine the separate and combined effects of speed and weight support on ground reaction forces (GRFs) and metabolic power during running Grabowski \& Kram, (2008), used a lower body positive pressure (LBPP) device called the G-trainer. They compared running at slower speeds and at normal weight with running faster with weight support and concluded that GRFs are reduced at all levels of weight support. Grabowski, 2010 found that manipulating speed and weight using the Alter- $\mathrm{G}^{\circledR}$ (newer version of the Alter- $\mathrm{G}^{\circledR}$ G-trainer) while walking, reduces GRFs but maintains cardio respiratory demand. In addition, when compared to normal weight walking, walking faster with body weight support elicited the same metabolic demand. She also concluded that the LBPP allowed for similar gait patterns to normal weight land walking, does not impede circulation and is more comfortable, adjustable and can be used for extended periods of time.

Additionally, other studies demonstrated favorable changes in physical activity levels, exercise tolerance with similar results in cardio respiratory responses compared to over-ground walking (Aaslund \& Moe-Nilssen, 2008; Cutuck, et al., 2006; Figueroa, Manning, \& Escamilla, 2011; Grabowski, 2010; Grabowski \& Kram, 2008; Raffalt, Howgaard-Hansen, \& Jensen, 2013). Another important finding in a study conducted by Raffalt, et al., (2013) is that the time to exhaustion on the LBPP treadmill increased significantly compared to a regular treadmill. This finding indicates a lower energy cost of running which may result in improved performance . Furthermore, an increase in $\mathrm{VO}_{2}$, heart rate, and ventilation as running speed increased was consistent with the findings of Grabowski and Kram (2008). This study confirms that the LBPP treadmill is an optimal training modality for rehabilitation and low-impact training for athletes. While these findings support anti-gravity training as having positive changes in health outcomes in healthy individuals, further investigation is required to assess its' effectiveness in exercise training in normal weight individuals. 
Therefore, the purpose of this study was to determine the exercise intensity that elicits maximal fat oxidation while walking on a body weight supported treadmill in untrained normal weight males.

\section{Methods}

\subsection{Subjects}

Fourteen men, age $23 \pm 2$, height, $1.7 \pm 0.07 \mathrm{~m}, 79.73 \pm 6.1 \mathrm{~kg}$, with a body fat percentage of $14.01 \pm 4.9 \%$ were recruited for this study. Physical characteristics of the study participants are summarized in Table 1. Each gave written informed consent after the experimental procedures were explained to them, which was by approved by William Paterson University's Institutional Review Board. To standardize the testing conditions and to insure the safety of the participants, the following pre-test instructions were given to each person before their first visit: (1) abstain from eating 12 hours before the test (2) abstain from consuming caffeine-containing products for a minimum of 12 to 24 hours before the test (3) abstain from strenuous exercise for at least 24 hours before the test, and (4) Consult the researcher on the potential use of any over the counter medication as some may affect resting or exercise heart rate and may affect test accuracy. Participants were considered non-athletic based on completion of their PAR-Q questionnaire.

Table 1. Descriptive Statistics

\subsection{General Design}

The participants attended the laboratory for two sessions separated by at least three but not more than seven days following the anthropometric and body density measurements. All sessions took place in the same laboratory where air temperature was $24.1 \pm 3^{\circ} \mathrm{C}$ and relative air humidity was $20 \pm 5 \%$.

\section{Session 1 - (Anthropometric Measurements and Body Density):}

Participants reported to the Human Performance Lab at William Paterson University where baseline measures of height, weight, waist and hip circumference and body density were measured. Body composition was determined by measuring body density using standard underwater weighing techniques. Session 2 and 3: Exercise Test: At both sessions, subjects were familiarized with the equipment and procedures. The subjects reported to the laboratory after a 12-hour overnight fast and at approximately the same time for each of the two sessions to avoid variations in their circadian rhythms. Resting blood pressure was recorded and then the subjects were prepped for ECG placement to determine resting and exercise heart rates. Subjects were then fitted with a mask connected to a metabolic cart to collect expired gases to determine $\dot{\mathrm{V}} \mathrm{O}_{2 \text { peak. }}$. Subjects were then instructed to put on the neoprene shorts, which were then zipped in to the treadmill aperture. Before the test, the air pressure in the chamber adjusted to apply the proper lifting force for each subject by way of a built in pressure feedback system. The treadmill tests were randomized where the subject walked on the AlterG ${ }^{\circledR}$ at either $100 \%$ or $75 \%$ of their body weight.

After the calibration process a warm-up of $2.0 \mathrm{mph}$ for 3 minutes at $100 \%$ body weight was performed. The participants, in random order $(100 \%$ or $75 \% \mathrm{BW})$ began to walk at $3.3 \mathrm{mph}$ at $0 \%$ grade. The speed was held constant for the duration of the test where the gradient increased every 3 minutes by $3 \%$ up to $15 \%$ grade. Heart rate (HR) and rating of perceived exertion (RPE) were recorded during the last 5 seconds of each stage and blood pressure was recorded during the last 30 seconds of each stage. The test was terminated if the participant experienced adverse signs or symptoms (ACSM, 2017) or requested to stop. Additionally, participants believed to have reached their true $\dot{\mathrm{V}} \mathrm{O}_{2 \max }$ if the following conditions existed: (a) a plateau in $\dot{\mathrm{VO}}_{2}$ (b) a heart rate within 5-10 beats of their age-predicted maximal heart rate (220-age x 0.85), (c) RPE of 18-20 and (d) a respiratory exchange ratio (RER) of 1.10. Finally, $\dot{\mathrm{VO}}{ }_{2 \text { peak }}$ is the highest value of $\dot{\mathrm{VO}}{ }_{2}$ the subject attained on the test. All subjects participated in two tests on two separate days separated by at least three but no more than seven days once the informed consent and PAR-Q were completed. The participants completed the other condition to determine peak fat oxidation (PFO), peak oxygen consumption $\left(\dot{\mathrm{V}}_{2 \text { peak }}\right)$, and respiratory exchange ratio (RER). 


\subsection{Statistical Analysis}

Differences between the two treadmill conditions (100\% BW \& 75\% BW) were assessed for the following dependent variables: Peak fat oxidation (PFO), peak oxygen consumption $\left(\dot{\mathrm{V}}_{2 \text { peak }}\right)$ and respiratory exchange ratio (RER) for the total amount of work done were performed using a paired samples $t$-test. Statistical analysis was performed using the SPSS IBM statistical package (v. 25, Chicago, IL). A Kolmogorov-Smirnov \& Shapiro Wilk test for normality was completed on all variables to determine homogeneity of variances.

\section{Results}

The results are presented as mean and standard deviation.

Results were analyzed using the statistical package SPSS version 25.0, Chicago, Illinois. Calculation of Cohen's d effect size values for $t$-tests were classified as small (0.2), medium (0.5) and large (>0.8). Sample size and effect size calculations for all dependent variables were analyzed in $\mathrm{G} *$ Power 3.1 (Faul, Erdfelder, Buchner, \& Lang, 2009). Kolmogorov-Smirnov \& Shapiro Wilk tests of homogeneity of variances were done on the dependent variables, Fat Oxidation (FO), Peak Oxygen Consumption ( $\left.\dot{\mathrm{VO}}_{2 \text { peak }}\right)$ and Respiratory Exchange Ratio (RER). The tests showed no significant differences between groups, indicating normality of distributions. To determine the difference between the two levels of the independent variable $(100 \% \mathrm{BW}$ and $75 \% \mathrm{BW})$ on the AlterG ${ }^{\circledR}$ antigravity treadmill, a paired samples $t$-test was conducted on PFO, $\dot{\mathrm{V}} \mathrm{O}_{2 \text { peak }}$ and RER. Table 2 summarizes the mean $\pm \mathrm{SD}$ values for 14 participants $\mathrm{PFO}, \mathrm{V}_{2 \text { peak }}$ and $\mathrm{RER}$ for each $\mathrm{BW}$ condition, which was obtained in the last minute of the last stage.

Table 2. Mean \pm SD values for peak HR, RPE and $\mathrm{VO}_{2}$ peak

\begin{tabular}{llll}
\hline & $100 \% \mathrm{BW}$ & $75 \% \mathrm{BW}$ & $p$ \\
\hline $\mathrm{FO}(\mathrm{g} / \mathrm{min})$ & $0.17 \pm 0.4$ & $0.14 \pm 0.3$ & 0.55 \\
$\mathrm{RER}$ & $0.89 \pm 0.1$ & $0.87 \pm 0.1$ & 0.46 \\
$\mathrm{VO}_{2 \text { peak }}(\mathrm{ml} / \mathrm{kg} / \mathrm{min})$ & $17 \pm 3.0$ & $13 \pm 4.0$ & $0.003 *$
\end{tabular}

Values are means \pm SD. FO, fat oxidation; RER, respiratory exchange ratio; $\mathrm{VO}_{\text {2peak }}$, peak oxygen consumption. $* p<0.01,100 \% \mathrm{BW}$ compared to $75 \% \mathrm{BW}$.

\subsection{Peak Fat Oxidation (PFO)}

As exercised intensity fat oxidation rates decreased from $0.28 \mathrm{~g} / \mathrm{min}$ to $0.15 \mathrm{~g} / \mathrm{min}$ for the $100 \% \mathrm{BW}$ and 0.22 $\mathrm{g} / \mathrm{min}$ to $0.14 \mathrm{~g} / \mathrm{min}$ at $75 \% \mathrm{BW}$. A paired samples $t$-test showed a difference in PFO rates between the $100 \% \mathrm{BW}$ condition and the $75 \% \mathrm{BW}$ condition were not statistically significant, $(t(13)=.61, p>.05), 95 \% C I(-.07,0.12)$, $d=0.82$. Figure 1 shows the difference between the two treadmill conditions where on average participants in the $100 \%$ BW group had lower fat oxidation rates $(M=-0.17, S D=0.36)$ than the $75 \%$ BW group $(M=0.14, S D=$ 0.27). Furthermore, fat oxidation rates peaked at $48 \%$ of $\dot{\mathrm{VO}}_{2 \text { peak }}(0.28 \mathrm{~g} / \mathrm{min}$ at $10.59 \mathrm{ml} / \mathrm{kg} / \mathrm{min}$, respectively) in the $100 \% \mathrm{BW}$ condition where fat oxidation rates peaked at $58 \%$ of $\dot{\mathrm{VO}}_{2 \text { peak }}(0.23 \mathrm{~g} / \mathrm{min}$ at $9.64 \mathrm{ml} / \mathrm{kg} / \mathrm{min})$ in the $75 \%$ BW condition (Figure 4).

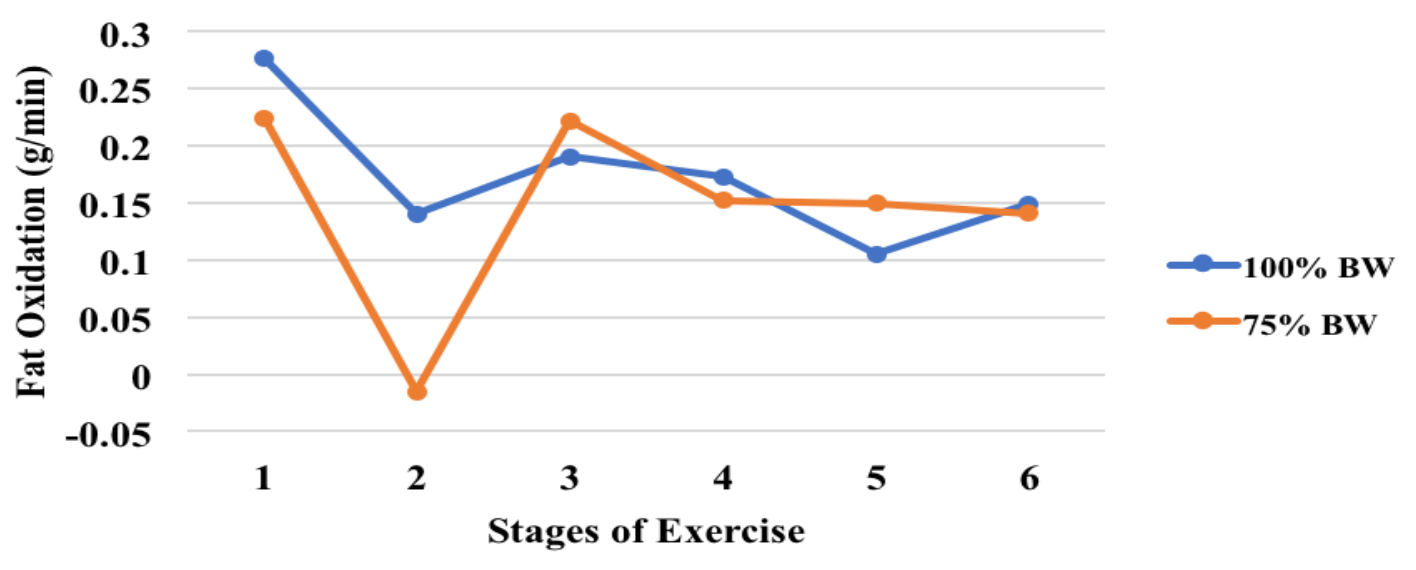

Figure 1. Rate of fat oxidation (g/min) at $100 \% \mathrm{BW}$ and $75 \% \mathrm{BW}$ as exercise intensity increases every three minutes. 


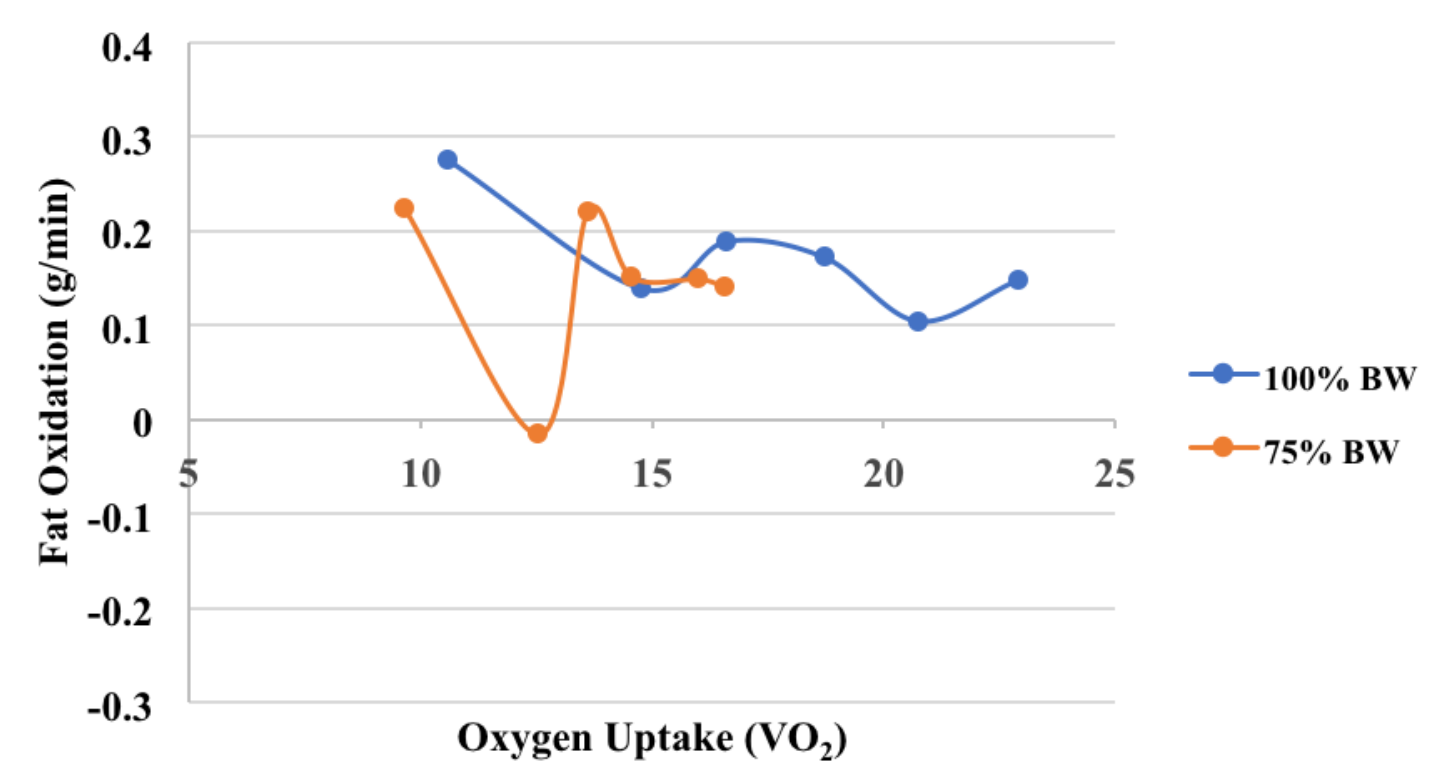

Figure 4. Graphic shows rates of peak fat oxidation relative to oxygen uptake for every three minutes of exercise.

\subsection{Peak Oxygen Consumption $\left(\dot{\mathrm{V}} \mathrm{O}_{2 \text { peak }}\right)$}

Oxygen consumption peaked at $22.89 \mathrm{ml} / \mathrm{kg} / \mathrm{min}$ in the $100 \%$ BW group and peaked at $16.55 \mathrm{ml} / \mathrm{kg} / \mathrm{min}$ in the $75 \%$ BW group. A paired samples $t$-test showed the difference in $\mathrm{VO}_{2 \text { peak }}$ between the $100 \% \mathrm{BW}$ and $75 \% \mathrm{BW}$ condition were statistically significant, $(t(13)=3.57, p<0.001), 95 \% C I(1.41,5.73), d=.81$. The effect size of 0.80 and was found to exceed Cohen's (1988) convention for a large effect size. Figure 2 shows the difference between the two treadmill conditions where participants in the $100 \% \mathrm{BW}$ group reached a higher $\mathrm{V}_{2 \text { peak }}(M=$ $17.38, S D=2.64)$ than the $75 \%$ BW group $(M=13.81, S D=3.59)$.

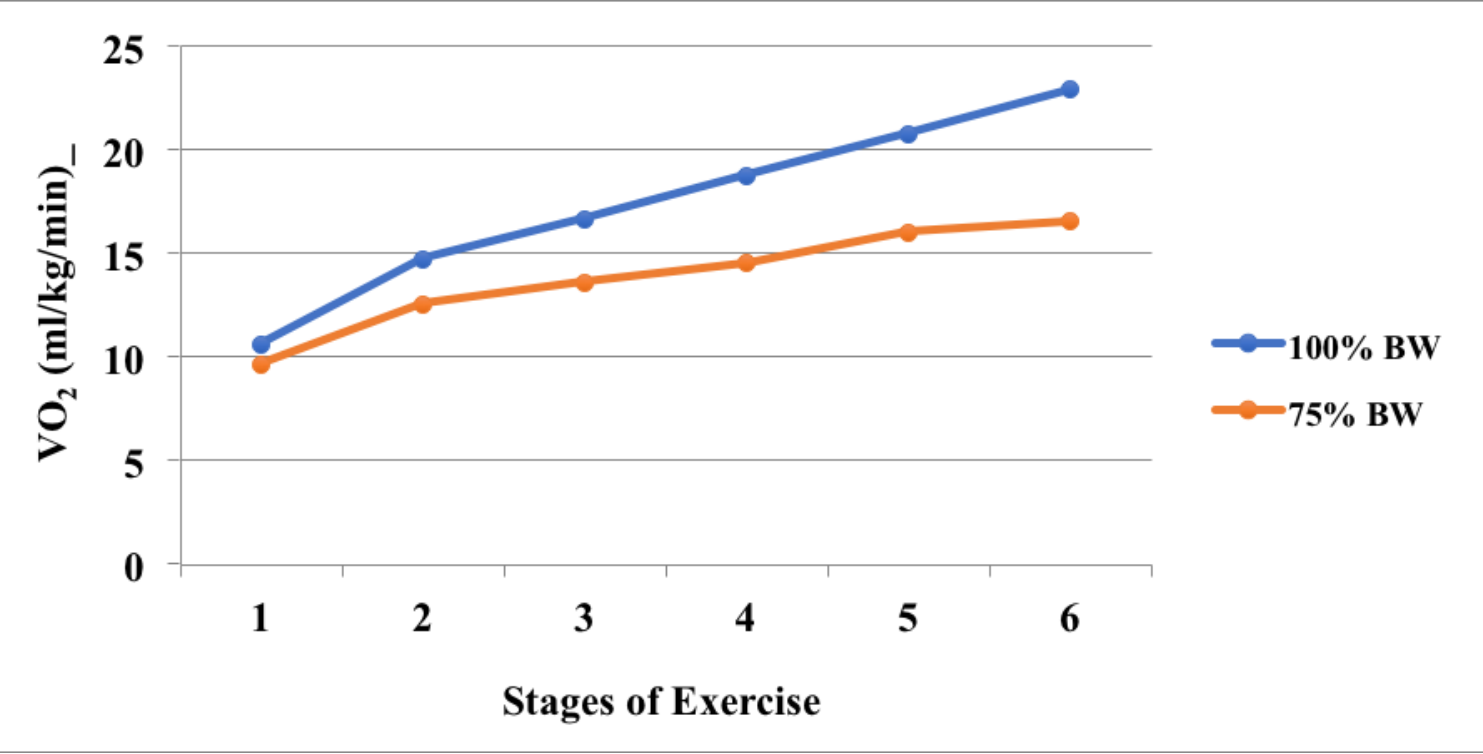

Figure 2. Oxygen consumption $\left(\mathrm{VO}_{2}\right)$ measured during each three minute stage of exercise on the AlterG ${ }^{\circledR}$ at $100 \% \mathrm{BW}$ and $75 \% \mathrm{BW}$. Graphic shows that as exercise intensity increases $\mathrm{VO}_{2}$ increases.

\subsection{Respiratory Exchange Ratio (RER)}

A paired samples $t$-test showed no significant difference in RER values averaged over the last minute of each stage between the $100 \%$ BW condition and the $75 \%$ BW condition, $(t(13)=0.72, p>0.05), 95 \% C I(.001,0.13), d$ $=.20$. The effect size of .20 is a small effect according to Cohen's (1988) convention for a small effect size $(d=0.20)$. 
Figure 3 shows the difference between the two treadmill conditions where participants in the 100\% BW group had higher RER's $(M=0.89, S D=0.07)$ than the $75 \%$ BW group $(M=0.87, S D=0.08)$. Furthermore, at $100 \%$ and $75 \%$ BW, PFO occurred in the first 3 minutes of exercise at an RER of 0.80 indicating a $66.6 \%$ contribution of fat.

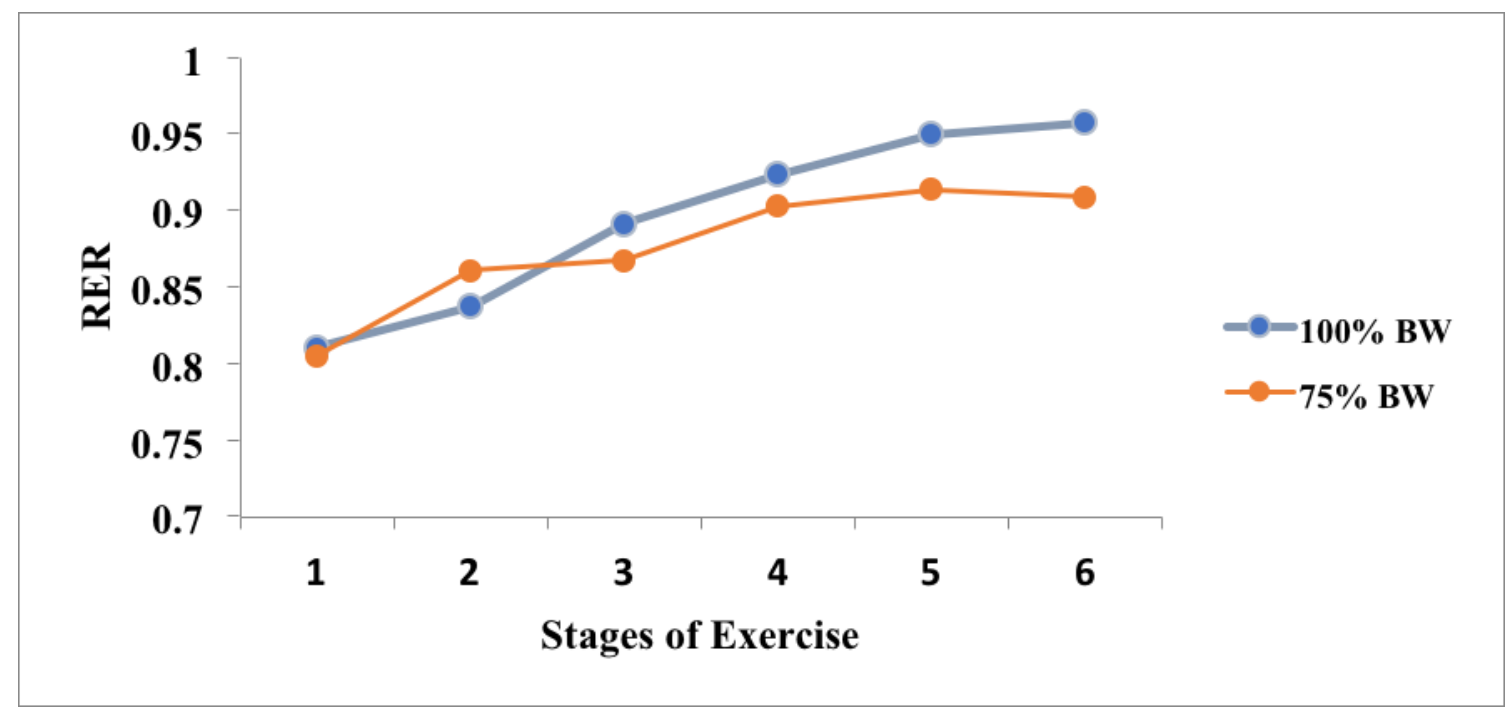

Figure 3. Respiratory exchange ratio (RER) measured on the AlterG $^{\circledR}$ at $100 \% \mathrm{BW}$ and $75 \% \mathrm{BW}^{\text {at }}$ each three-minute stage of exercise.

\section{Discussion}

The purpose of the present study was to determine the exercise intensity that elicits maximal or peak fat oxidation while walking on a body weight supported treadmill at $100 \% \mathrm{BW}$ and $75 \% \mathrm{BW}$. We also looked at the percentage of $\dot{\mathrm{VO}}_{2 \text { peak }}$ at which PFO occurred as well as RER in untrained normal weight males. The maximal rate of fat oxidation (FO) during exercise typically occurs at moderate intensities of 45 to $65 \%$ of maximal oxygen consumption. The main finding of this study was that fat oxidation rates in the decreased $\mathrm{BW}$ condition, was observed at a moderate intensity $\left(58 \% \dot{\mathrm{VO}}_{2 \text { peak }}\right)$ compared to the $100 \% \mathrm{BW}$ condition at $47 \% \dot{\mathrm{VO}}_{2 \text { peak }}$, which is also considered moderate.

Exercise intensity is one of the most important factors that determine fat oxidation rates during exercise. Exercise intensity in most studies is expressed as a percentage of $\dot{\mathrm{VO}}_{2 \max }$. In a study on fat oxidation rates over a wide range of intensities, maximal rates of fat oxidation ranged from approximately 47 to $52 \% \mathrm{VO}_{\max }$ in the general population (Achten \& Jeukendrup, 2004). Furthermore, MFO has also been found at low to moderate intensities that range from 33 to $65 \%$ of $\dot{\mathrm{VO}}_{2 \max }$ (Romijn et al., 1993; Friedlander et al., 1998; \& van Loon et al., 2001; Venables, Achten \& Jeudendrup, 2005). Optimal intensity for fat oxidation is approximately $50 \%$ of $\dot{\mathrm{VO}}_{2 \max }$ for untrained individuals (Achten \& Jeukendrup, 2004; Jeukendrup \& Wallis, 2005) where activities of intensities higher than MFO have shown that FO rates decrease markedly (Achten et al., 2002).

Fat oxidation rates in the current study peaked at $47 \%$ of the subjects $\dot{\mathrm{V}} \mathrm{O}_{2 \text { peak }}(0.28 \mathrm{~g} / \mathrm{min}$ at $10.59 \mathrm{ml} / \mathrm{kg} / \mathrm{min})$, which occurred in the first three minutes of the exercise session (warm-up) at 100\% BW and then decreased markedly at minute six as exercise intensity increased at $64 \%$ of $\dot{\mathrm{V}} \mathrm{O}_{2 \text { peak }}(0.014 \mathrm{~g} / \mathrm{min}$ at $14.75 \mathrm{ml} / \mathrm{kg} / \mathrm{min})$ which is consistent with other studies (Achten \& Jeukendrup, 2004; Romijn et al., 1993; Friedlander et al., 1998; \& van Loon et al., 2001; Venables, Achten \& Jeudendrup, 2005; Jeukendrup \& Wallis, 2005). At the third stage or minute nine, up to the end of the exercise session fat oxidation rates increased slightly then began to decrease as exercise intensity increased. In the $75 \% \mathrm{BW}$ condition fat oxidation rates peaked at $58 \%$ of the subjects $\mathrm{V}_{2 \text { peak }}$ $(0.23 \mathrm{~g} / \mathrm{min}$ at $9.64 \mathrm{ml} / \mathrm{kg} / \mathrm{min})$ which occurred in the first three minutes of the exercise session. This was greater than the rate of fat oxidation seen in the $100 \% \mathrm{BW}$ condition in the same time frame, which cannot be explained. However, peak fat oxidation rates in the current study at $100 \%$ and $75 \% \mathrm{BW}\left(46 \%\right.$ to $58 \%$ of $\mathrm{VO}_{2 \text { peak }}$ ) are consistent with other studies done on fat oxidation (33\% to $65 \% \mathrm{VO}_{2 \text { peak }}$ ) (O'Deriaz et al. 2001; Bogdanis et al. 2001; Romijn et al., 1993; Friedlander et al., 1998; \& van Loon et al., 2001; Venables, Achten \& Jeudendrup, 2005; van Aggel-Liejssen et al., 2002). 
These values are equivalent to an exercise range that is considered moderate in intensity, one which is currently suggested by the American College of Sports Medicine for those individuals interested in weight control and cardiovascular health (ACSM, 2017).

Exercise training and substrate utilization is well documented in normal weight individuals (Jeukendrup et al., 1997; Kanaley et al., 2001; van Loon et al., 1999), where fat is the preferred energy source at rest and low to moderate intensity exercise of long duration (Brooks \& Mercer, 1994; O’Brien et al., 1993; Romijn et al., 1993; Thompson et al., 1998). Sial et al., (1999) demonstrated that exercise training in elderly subjects increased total fat oxidation without a change in lipolysis or free fatty acid (FFA) availability. It is suggested based on past and current research, to maintain the \% gradient at the level of maximal fat oxidation (3\% grade or less) and perhaps more fat would be oxidized over a longer period of time.

RER is the ratio of carbon dioxide $\left(\mathrm{CO}_{2}\right)$ produced to oxygen $\left(\mathrm{O}_{2}\right)$ consumed, which is an indicator of which fuel is being oxidized. According to Lusk (1928), an RER of 0.71 indicates $100 \%$ fat oxidation. In the current study, PFO occurred in the first three minutes at an RER of $0.81 \%$ in the $100 \% \mathrm{BW}$ condition indicating $66.6 \%$ fat oxidation. In the $75 \% \mathrm{BW}$ condition, PFO also occurred in the first three minutes at an RER of 0.8 , which also indicates $66.6 \%$ fat oxidation.

RER increased from 0.81 to 0.9 in both conditions suggesting the majority of fat oxidation occurred in the first three minutes or the warm-up phase of the protocol. Although RER increased significantly as exercise intensity increased the overall message is that exercise intensity needs to be consistently lower throughout the protocol. The speed in the current study remained constant, so it would be suggested to decrease the percent grade to maintain elevated levels of fat oxidation.

In the current study there were no significant differences in fat oxidation between both BW conditions, which cannot be explained. However, other studies have shown that total fat oxidation during low intensity exercise was greater in obese than in normal weight individuals (Horowitz \& Klein, 2000; Kanaley et al., 2001; \& Goodpaster et al., 2002). In contrast, fat oxidation at different intensities in overweight/obese individuals showed a lower rate of fat oxidation than normal weight individuals (Perez-Martin et al., 2001; Bogdanis et al., 2005). In the present study, those who were in the reduced BW group showed no significant difference in fat oxidation at a lower intensity, which then began to decrease as intensity increased. Different body weights, distribution of body fat, current fitness level and diet may affect substrate utilization during exercise. Differences were significant in $\dot{\mathrm{VO}}_{2 \text { peak }}$, which demonstrated that anyone with physical limitations could still benefit from LBPP training in an unweighted condition.

It has been shown that fat oxidation rates can be reproduced in one individual; however several studies indicate there is a large inter-individual variation as to where maximal fat oxidation occurs. A cross sectional study demonstrated that large differences exist in the ability to oxidize fat during exercise (Venables et al., 2005). In the same study, fat oxidation rates were shown to range from 0.18 to $1.01 \mathrm{~g} / \mathrm{min}$. They concluded that the lean body mass, physical activity levels, $\mathrm{VO}_{2 \max }$, gender and fat mass accounted for $35 \%$ of the variation in peak fat oxidation rates, however $66 \%$ of the variance could not be explained. Although diet is likely to explain some the variance there is still a large part of the variance that remains unexplained.

\section{Conclusion}

Fat oxidation is important in controlling body weight in both trained and untrained individuals. In a recent metaanalysis, it is well confirmed that training at maximal fat oxidation ( 3 times per week) decreases fat mass, body weight and improves cholesterol (Romain et al., 2012). Endurance exercise training and regular physical activity have been shown to increase total energy expenditure and lipid oxidation at rest (Blaak \& Saris, 2002; Schrauwen, van Aggel-Leijssen, Hul, 2002) in healthy lean subjects. Unweighting demonstrated that the exercise intensity that promoted maximal fat oxidation occurred at $58 \% \dot{\mathrm{VO}}_{2 \text { peak. }}$.

The results of the present investigation provide practical implications for an optimal exercise prescription utilizing the anti-gravity treadmill. As indicated in other studies, there also seems to be a large variation where peak fat oxidation occurs at a given intensity and has been suggested that this is a result of body composition, gender, and the type of exercise activity (Achten et al., 2003, Perez-Martin et al., 2001; Tarnopolsky, MacDougall, Atkinson, Tarnopolsky, \& Sutton 2008). 
There is also emerging evidence that LBPP devices appear to have a variety of benefits with no harmful effects in men, women and the elderly (Cutuk et al., 2006). Therefore, LBPP training is a modality that is gaining popularity to study unweighting the body during exercise without altering gait, heart rate or blood pressure. In conclusion, if the goal when prescribing exercise to a normal weight population is to increase fat oxidation then based on the results of the present investigation, it would be suggested to unweight the body to $75 \%$ at an exercise intensity that yields a maximal fat oxidation rate at $58 \%$ of $\mathrm{VO}_{2 \text { peak }}$ with a percent grade of $3 \%$ or less.

\section{References}

Aaslund, M.K., \& Moe-Nilssen, R. (2008). Treadmill walking with body weight support effect of treadmill, harness and body weight support systems. Gait \& Posture, 28, 303-308.

Achten J., Gleeson, M., \& Jeukendrup, A.E. (2002). Determination of the exercise intensity that elicits maximal fat oxidation. Medicine Science Sports Exercise 34, 92-97.

Achten, J. \& Jeukendrup, A. E. (2003). Maximal fat oxidation during exercise in trained men. International Journal of Sports Medicine, 24, 603-608.

Achten, J., \& Jeudendrup, A.E. (2004). Optimizing fat oxidation through exercise and diet. Nutrition, 20, 716727.

American College of Sports Medicine. (2017). ACSM's guidelines for exercise testing and prescription (10th ed.). Baltimore, MD; Lippincott Williams and Wilkins.American Physiological Society, 2005

Bogdanis, G.C., Vangelakoudi, A.,\& Maridaki, M. (2008). Peak oxidation rate during walking in sedentary overweight men and women. Journal of Sports Science and Medicine, 7, 525- 531.

Brooks, G.A. \& Mercier, J. (1994). Balance of carbohydrate and lipid utilization during exercise:the "crossover" concept. Journal of Applied Physiology, 76, 2253-2261.

Brun, J-F., Varlet-Marie, E., Romain, A.J. \& Mercier, J. (2012). Measurement and physiological relevance of the maximal lipid oxidation rate during exercise (LIPOmax). An International Perspective on Topics in Sports Medicine and Sports Injury, Dr. Kenneth R. Zaslaw (Ed.), Retrieved from:

http://www.intechopen.com/books/an-international-perspective-on-topics-in-sports-medicine-and-sportsinjury/measurement-and-physiological-relevance-of-the-maximal-lipid-oxidation-rate-during-exerciselipomax

Centers for Disease Control. (2016). Retrieved from http://www.cdc.gov/obesity/data/index.html

Cutuk, A., Groppo, E.R., Quigley, E.J., White, K.W., Pedowitz, R. A., \& Hargens, A.R. (2006). Ambulation in simulated fractional gravity using lower body positive pressure: cardiovascular safety and gait analysis. Journal of Applied Physiology, 101(3), 771-777. doi:10.1152/japplphysiol.00644-2005.

Faul, F., Erdfelder, E., Buchner, A., \& Lang, A.-G. (2009). Statistical power analyses using G*Power 3.1: Tests for correlation and regression analyses. Behavior Research Methods, 41, 1149-1160.

Figueroa, M.A., Manning, J., \& Escamilla, P. (2011). Physiological responses to the AlterG® anti-gravity treadmill. International Journal of Applied Science and Technology,1 (6), 92-97.

Grabowski, A. (2010). Metabolic and biomechanical effects of velocity and weight support using alower body positive pressure device during walking. Archives of Physical Medicine and Rehabilitation, 91, 951-957.

Grabowski, A., \& Kram, R. (2008). Effects of velocity and weight support on ground reaction forces andmetabolic power during running. Journal of Applied Biomechanics, 24, 288- 297.

Grabowski, A., Farley, C.T., \& Kram, R. (2005). Independent metabolic cost of supporting body weight and accelerating body mass during walking. Journal of Applied Physiology, 98, 579- 583. doi:10.1152/japplphysiol.00734.2004.

Griffin, T.M., Tolani, N.A., \& Kram, R. (1999). Walking in simulated reduced gravity: mechanical energy fluctuations and exchange. Journal of Applied Physiology, 86, 383-390.

Doi: 10.1152/jappl.1999.86.1.383

Jeukendrup, A. E. (2003). Modulation of carbohydrate and fat utilization by diet, exercise and environment. Biochemical Society Transactions, 31, 1270-1273.

Jeukendrup, A. E., Mensink, M., Saris, W. H., \& Wagenmakers, A. J. (1997). Exogenous glucose oxidation during exercise in endurance-trained and untrained subjects. Journal of Applied Physiology, 82, 835840.

Jeukendrup, A. E., Saris, W. H., \&Wagenmakers, A. J. (1998). Fat metabolism during exercise: a review. Part I: Fat mobilization and muscle metabolism. International Journal of Sports Medicine, 19, 231-244. 
Jeukendrup, A. E., \& Wallis, G. A. (2005). Measurement of substrate oxidation during exercise by means of gas exchange measurements. International Journal of Sports Medicine, 26, 28-37.

Kanaley, J.A., Weatherup-Dentes, M.M., Alvarado, C.R., \& Whitehead, G. (2001). Substrate oxidation during acute exercise and with exercise training in lean and obese women. European Journal of Applied Physiology. 85, 68-73.

Nordby, P., Saltin, B., \& Helge, J. W. (2006). Whole-body fat oxidation determined by graded exercise and indirect calorimetry: a role for muscle oxidative capacity? Scandinavian Journal of Medicine \& Science in Sports, 16 (3), 209-214.

O’Deriaz, M., Dumont, M., Bergeron, N., Despres, J. P., Brochu, M., \& Prud'homme, D. (2001). Skeletal muscle low attenuation area and maximal fat oxidation rate during submaximal exercise in male obese individuals. International Journal of Obesity, 25 (11), 1579-1584.

Perez-Martin, A., \& Mercier, J. (2001). Stress tests and exercise training program for diabetics-initial metabolic evaluation. Annales D Endocrinologie, 62 (4), 291-293.

Raffalt, P.C., Howgaard-Hansen, L., \& Jensen, B.R. (2013). Running on a lower-body positive pressure, Peak VO2, respiratory response, and vertical ground reaction force. Research Quarterly for Exercise and Sport, 84, 213-222.

Romijn, J.A., Coyle, E.F., Sidossis, L.S., Gastaldelli, A. Horowitz, J.F., Endert, E. \& Wolfe, R.R. (1993). Regulation of endogenous fat and carbohydrate metabolism in relation to exercise intensity. American Journal of Physiology, 265, 380-391.

Schrauwen, P., van Aggel-Leijssen, D.P. \& Hul, G. (2002). The effect of a 3-month low-intensity endurance training program on fat oxidation and acetyl-CoA carboxylase-w expression. Diabetes, 51, 2220-2226.

Sial, S., Coggan, A. R., Hickner, R. C., \& Klein, S. (1998). Training-induced alterations in fat and carbohydrate metabolism during exercise in elderly subjects. American Journal of Physiology, 274, E785-E790.

Takagi S, Sakamoto S, Midorikawa T, Konishi M, Katsumura T. (2014). Determination of the exercise intensity that elicits maximal fat oxidation in short-time testing. Journal of Sport Science, 32, 175-182.

Tarnopolsky, L.J., MacDougall, J.D., Atkinson, S.A., Tarnopolsky, M.A., \& Sutton, J.R. (1990). Gender differences in substrate for endurance exercise. Journal of Applied Physiology, 68, 302-308.

Thompson, D.L., Townsend, K.M., Boughey, R., Patterson, K., \& Bassett Jr., B.R. (1998). Substrate use during and following moderate and low-intensity exercise: Implication for weight control. European Journal of Applied Physiology, 78, 43-49.

vanAggel-Leijssen, D.P., Saris, W.H., Wagenmakers, A.J., Hul, G.V., \& van Baak, M.A. (2001). The effect of low-intensity exercise training on fat metabolism of obese women. Obesity Research, 9, 86-96.

vanAggel-Leijssen, D.P., Saris, W.H., Wagenmakers, A.J., Senden, J.M., \& van Baak, M.A. (2002). Effect of exercise training at different intensities on fat metabolism of obese men. Journal of Applied Physiology, 92, 1300-1309.

van Loon, J. L., Jeukendrup, A. E., Saris, W. H., \& Wagenmakers, A. J. (1999). Effects of training status on fuel selection during submaximal exercise with glucose ingestion. Journal of Applied Physiology, 87, 14131420.

Venables, M.C., \& Jeudendrup, A.E. (2008). Endurance training and obesity: Effect on substrate metabolism and insulin sensitivity. Medicine Science in Sports and Exercise, 40, 495-502.

Venables, M.C., Achten, J. \& Jeudendrup, A.E. (2005). Determinants of fat oxidation during exercise in healthy men and women: A cross-sectional study. Journal of Applied Physiology, 98, 160-167.

Zurlo, F., Lillioja S., Puente, E.A., Nyomba, B.L., Raz I, Saad, M.F., Swinburn, B.A., Knowler W.C., Bogardus, C., Ravussin E. Low ratio of fat to carbohydrate metabolism as predictor of weight gain: study of 24-h RQ. American Journal of Physiology and Endocrine Metabolism 259, E650 -E657, 1990. 